EPJ Web of Conferences 52, 09006 (2013)

DOI: 10.1051/epjconf/20135209006

(C) Owned by the authors, published by EDP Sciences, 2013

\title{
Atmospheric Muons as IceCube Signal
}

\author{
Patrick Berghaus $^{1, a}$ for the IceCube Collaboration ${ }^{\text {b }}$ \\ ${ }^{1}$ DESY-Zeuthen, Platanenallee 6, D-15738 Zeuthen, Germany
}

\begin{abstract}
Muons of energies above $1 \mathrm{TeV}$ produced in cosmic ray induced air showers account for the vast majority of events in IceCube. Its enormous size compared to previous volume detectors translates into an unprecedented amount of statistics for high-energy atmospheric muons. This offers a wide range of opportunities for original cosmic ray and particle physics.

By identifying highly energetic stochastic losses within the detector volume, the single muon spectrum can be measured up to $\mathrm{PeV}$ energies. The result is sensitive to the cosmic ray composition around the knee and the contribution to atmospheric lepton fluxes from prompt hadron decays. The multiplicity spectrum of muon bundles relates to the cosmic ray primary flux and composition. Clear features are visible, which can be used to constrain phenomenological models. Investigation of high- $p_{T}$ muons at previously inaccessible lateral separations point to shortcomings in current hadronic interaction models. Furthermore, the large event statistics allow detailed investigation of anisotropies in the arrival direction of cosmic rays for primary energies in excess of 1 $\mathrm{PeV}$.
\end{abstract}

\section{Introduction}

The main purpose of the IceCube deep detector is the search for astrophysical neutrinos [1]. However, the actual event yield consists almost exclusively of muons produced in atmospheric showers. Understanding this data is essential for evaluation of detector performance and represents a valuable opportunity for research into previously inaccessible areas of cosmic ray and particle physics.

The main asset of IceCube, apart from its sheer size, is the three-dimensional extension of its instrumented volume. Its obvious shortcoming is the lack of sensitivity to the electromagnetic part of air showers, generally restricting its sensitivity to $\mathrm{TeV}$ muons energetic enough to penetrate several kilometers of ice. Coincident events between the surface array IceTop [2] and the deep detector are available only for a relatively small angular region around the zenith.

In practice this means that analyses related to cosmic rays with the deep detector alone only have access to the leptonic part of the shower, and primary energy and mass cannot be deconvoluted. Energy spectra can be constructed, but will typically be degenerate with the nuclear composition. This constraint is partially offset by the full angular coverage and the strong seasonal variations of the Antarctic atmosphere, which can be exploited to identify the individual components of the atmospheric lepton flux from pion, kaon, and prompt hadron decays [3].

While exclusive reliance on the high-energy muons component of air showers represents a limitation, this is

\footnotetext{
a e-mail: berghaus@icecube.wisc.edu

$\mathrm{b}_{\text {author list: http://icecube.wisc.edu/collaboration/authors/current }}$
}

more than compensated by the broad energy spectrum covered by the instrument. The sensitivity of IceCube to cosmic rays reaches from approximately $10 \mathrm{TeV}$ to $10 \mathrm{EeV}$, where the lower limit is determined by the effective production threshold for $\mathrm{TeV}$ muons, and the upper by the flux of cosmic ray primaries. Neutrino interactions, with a threshold of few tens of $\mathrm{GeV}$, extend this range even further.

\section{Muon Multiplicity Spectrum}

The number of muons in an air shower above a given energy $E_{\mu}$, produced by a primary of mass $A$ and energy $E_{\text {prim }}$, can be approximated as

$$
N_{\mu}\left(E>E_{\mu}\right)=A \cdot \frac{E_{0}}{E_{\mu} \cos \theta} \cdot\left(\frac{E_{\text {prim }}}{A E_{\mu}}\right)^{\alpha} \cdot\left(1-\frac{A E_{\mu}}{E_{\text {prim }}}\right)^{\beta}
$$

where $\alpha=0.757, \beta=5.25$ and $E_{0}=14.5 \mathrm{GeV}$ were determined by a numerical simulation [4]. For energies not too close to the production threshold $E_{\text {prim }} / A$, this relation can be simplified to:

$$
N_{\mu} \propto A^{1-\alpha} \cdot E_{p r i m}^{\alpha}
$$

For deep muon detectors, the $E_{\mu}$ is determined by the threshold energy for muons penetrating the ice above the detector. For vertical events, this corresponds to about 400 $\mathrm{GeV}$, increasing exponentially as a function of $\sec \theta_{z e n}$. Using air shower simulations, it can be demonstrated that in the case of IceCube Eq. 2 is valid to about 20 percent at 

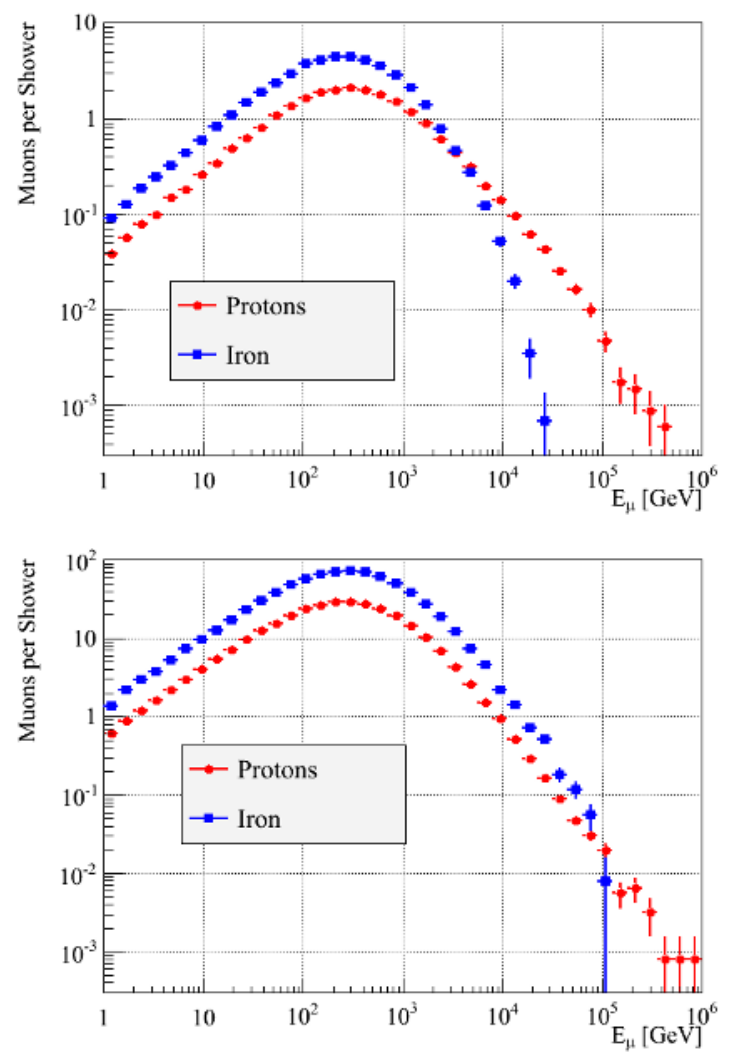

Figure 1. Distribution of muon energies in individual air showers at the IceCube detector depth simulated with CORSIKA/SIBYLL. Top: $E_{\text {prim }}=3 \mathrm{PeV}$. Bottom: $E_{\text {prim }}=100 \mathrm{PeV}$. The threshold effects visible at high muon energies in the top plot are due to the lower energy per nucleon in iron. As the total energy increases, this effect becomes less and less visible and the spectra are identical except for a scaling factor.

primary energies of $1 \mathrm{PeV}$, and rapidly improving as the energy increases.

Equation 1 furthermore implies that the distribution of muon energies within a shower is independent of the primary type and energy, except at the very highest end of the spectrum. Full shower simulations support this conclusion, as illustrated in Fig. 1. The total energy of the muon bundle, as well as its energy loss per unit track length, is therefore in good approximation simply proportional to the muon multiplicity.

It is convenient to define a parameter

$$
E_{\text {mult }} \equiv E_{\text {prim }} \cdot(A / 56)^{\frac{1-\alpha}{\alpha}}
$$

which accounts for the implicit degeneracy between primary energy and mass. The scaling has been chosen such that $E_{\text {mult }}$ is equal to $E_{\text {prim }}$ in the case of iron primaries, which will in practice account for the dominant part of the flux spectrum, as demonstrated in Fig. 2. This parameter can then be related to the muon multiplicity in the detector $N_{\mu, d e t}$ as

$$
E_{\text {mult }}=\eta(\cos \theta) \cdot N_{\mu, d e t}^{1 / \alpha}
$$
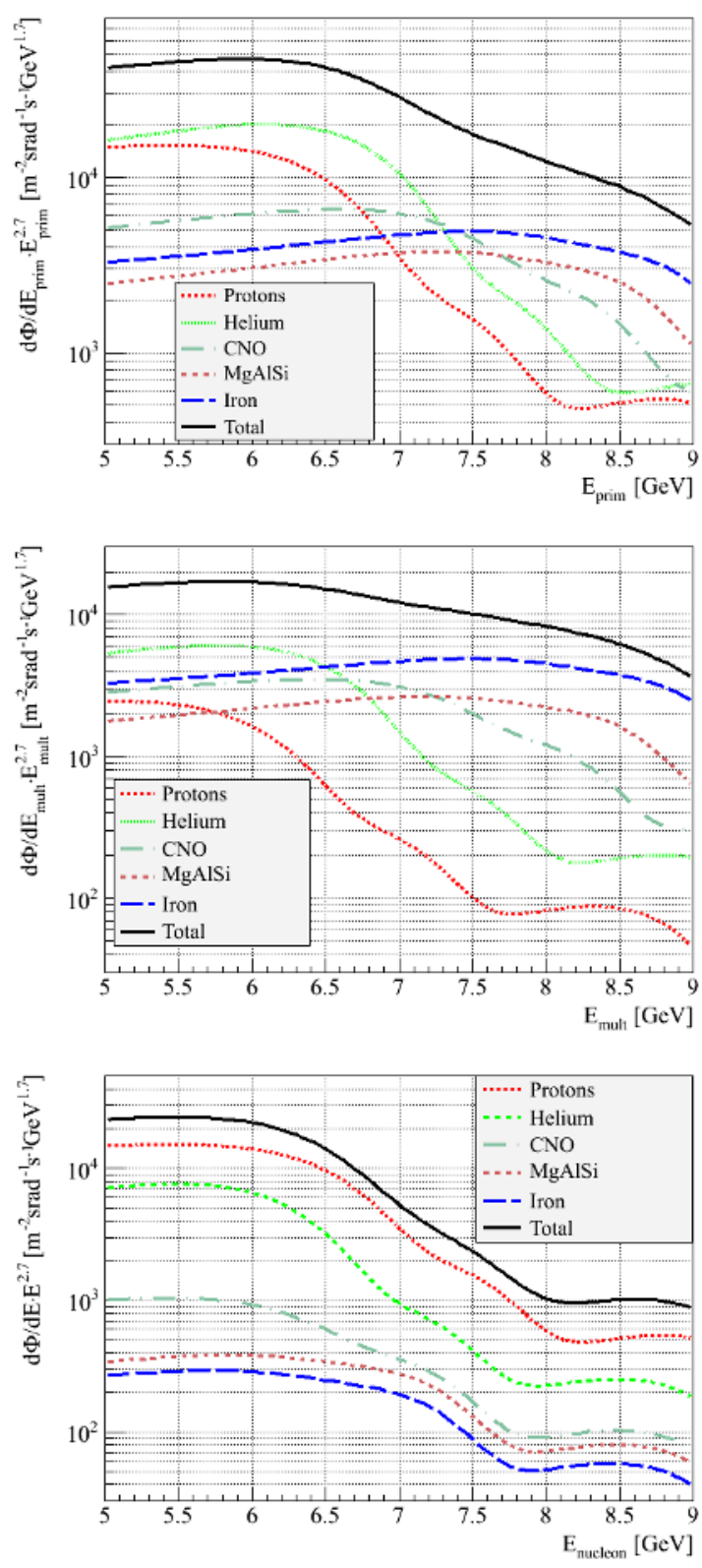

Figure 2. Comparison of primary energy (top), rescaled muon multiplicity parameter $E_{\text {mult }}$ (middle, definition see text) and nucleon energy (bottom) spectra for Hillas model with mixedcomposition extragalactic component as described by Gaisser [5]. For a flux of spectral index $\gamma$, the contribution of primaries of mass $A$ to the muon multiplicity scales as $\frac{\Phi_{\text {mult }}}{\Phi_{\text {prim }}} \simeq A^{\frac{1-\alpha}{\alpha} \cdot(\gamma-1)}$, with $\alpha \approx 0.76$. By construction, $E_{\text {mult }}=E_{\text {prim }}$ for Fe primaries. The corresponding relation for the nucleon spectrum is $\frac{\Phi_{n u c}}{\Phi_{\text {prim }}} \simeq A^{2-\gamma}$.

where $\eta(\cos \theta)$ is a simulation-derived function accounting for angular dependence of muon production and absorption in the surrounding material. Using the above definition, experimental data can be directly related to any flux model expressed as a function of $E_{\text {mult }}$. After excluding rare events where the muon energy deposition is dominated by exceptional catastrophic losses as illustrated by Fig. 3, the muon multiplicity can be measured without requiring more than purely calorimetric information. 


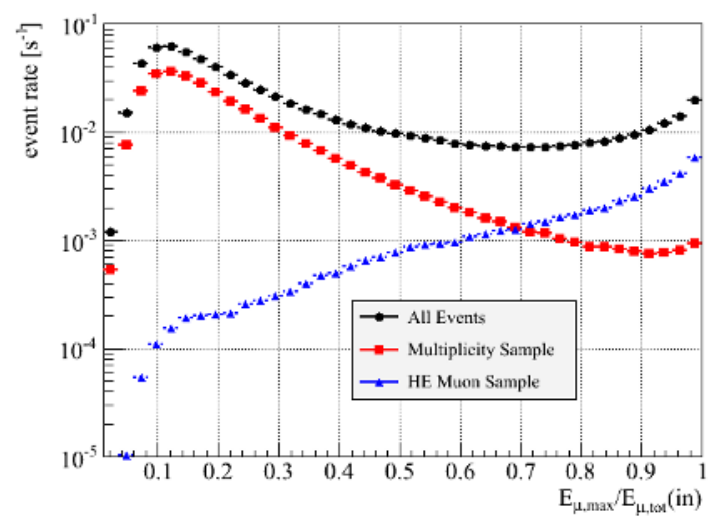

Figure 3. Simulated rates of events with more than 1,000 registered photo-electrons for 79-string IceCube detector in dependence of fraction of bundle energy carried by the leading muon. The red curve represents the event sample used in the multiplicity analysis described in Section 2. Events in which the bundle is dominated by individual muons are suppressed, as they may lead to a distortion of the measurement. The blue curve describes the event sample used to derive the single muon energy spectrum as discussed in Section 3. The comparatively low efficiency is due to the necessity for an exceptionally strong stochastic loss to occur within the detector volume.

The IceCube detector consists of more than five thousand digital optical moduled (DOMs), each containing one photo-multiplier tube (PMT) [6]. The signal registered by the PMTs is digitized in situ and transmitted digitally to the surface [7].

The detection principle is based on the emission of Cherenkov light by highly energetic charged particles passing through the surrounding medium, glacial ice containing varying amounts of impurities. The number of emitted photons is in good approximation proportional to the energy loss. Neglecting effects due to local ice properties, the total amount of photo-electrons registered by the DOMs in a given event can therefore be used as a calorimetric observable. The zenith angle of the incident shower can be determined by a standard likelihood-based track reconstruction.

Due to bandwidth limitations, only selected IceCube events are transmitted to the northern hemisphere. A complete unbiased sample for the 79-string detector configuration is only available for events with a total of more than one thousand registered photo-electrons. This threshold coincides approximately with energies close to $1 \mathrm{PeV}$, where threshold effects in heavy nuclei become important. For the analysis of high-multiplicity bundles as described here, the availability of events is therefore not a seriously limiting factor.

The relation between the number of detected photons and the actual muon multiplicity can be determined by a Monte-Carlo simulation. Using Eq. 4, the experimental value of $E_{\text {mult }}$ can thus be calculated. The resolution of the muon multiplicity estimator lies at about $0.15 \log _{10} N_{\mu}$ for bundles with tens of muons and improves to 0.1 at higher multiplcities, as shower fluctuations become less impor-

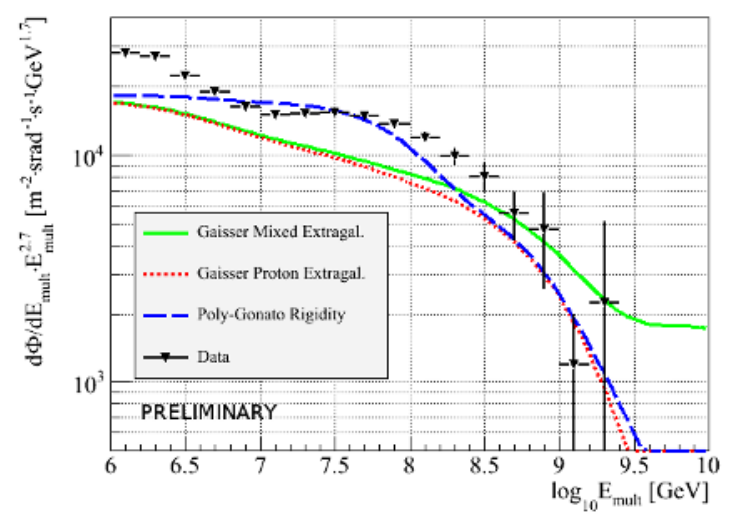

Figure 4. Rescaled muon multiplicity spectrum from one month of 79-string IceCube data compared to parametrized cosmic ray flux models [5][11]. For definition of $E_{\text {mult }}$, see text. In the experimental data, the muon multiplicity was estimated based on the total amount of photo-electrons registered for a given event. The difference between the two Gaisser models is due to proton primaries producing muon bundles of significantly lower multiplicities. Given enough statistics, it will be possible to constrain extragalactic flux models using muon data alone. Note that the Poly-Gonato model does not contain an extragalactic component. Errors on the data do not include systematic effects.

tant. The resolution of the experimental $E_{\text {mult }}$ proxy observable varies from 0.3 in $\log _{10}$ at $1 \mathrm{PeV}$ to 0.2 above 100 $\mathrm{PeV}$. Not included in this estimate are systematics uncertainties such as conversion of energy loss to photo-electron yield, angular acceptance of the DOMs, seasonal variations of the atmosphere, hadronic interaction models in the air shower simulation, limited knowledge of the ice properties and higher order biases depending on the primary composition.

The preliminary result, shown in Fig. 4, suggests a similar structure of the cosmic ray flux as recently measured by KASCADE-Grande and TUNKA [9][10]. Notably, the spectrum of $E_{\text {mult }}$ has a similar shape, but overall a harder spectral index by about 0.2 . The obvious interpretation would be an increase in average primary mass at high energies, consistent with expectation.

A detailed evaluation of the result on its own is challenging. However, in combination with measurements of energy spectrum and composition using the surface array IceTop, it should allow to model the cosmic ray flux above the knee with unprecedented precision. The accessible energy range reaches well into the ankle region where the transition to an extragalactic flux is expected to occur, as illustrated by Fig. 5 .

\section{Muon Energy Spectrum}

In contrast to the muon bundle multiplicity discussed in the previous section, the single muon energy follows the nucleon spectrum rather than the total primary flux. Since the energy per nucleon is significantly lower for heavy nuclei, only proton and helium primaries make a significant contribution, as illustrated in Fig. 2. 


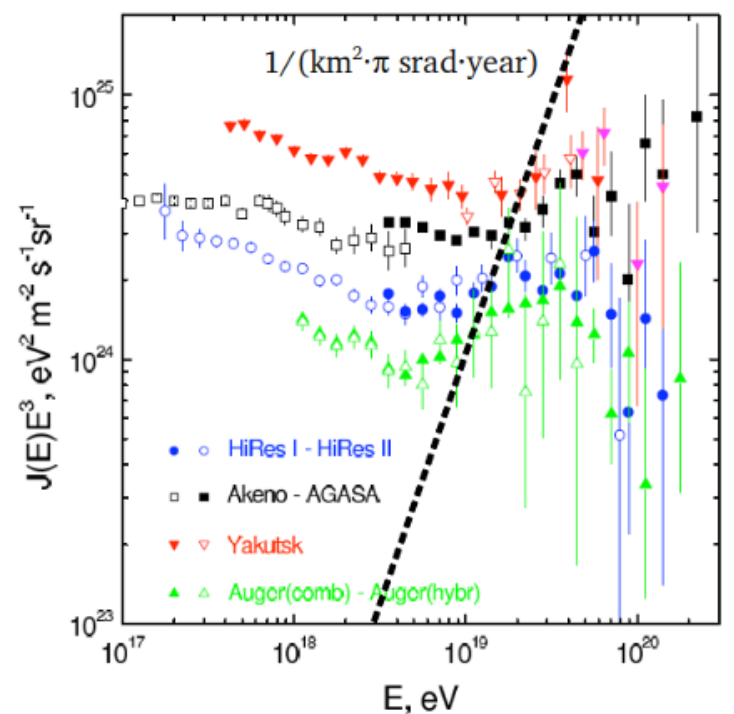

Figure 5. Primary cosmic ray flux rate in the ankle region measured by four different detectors [8]. The dashed curve corresponds to the approximate exposure in the muon multiplcity analysis (one event per $\mathrm{km}^{2} \cdot \pi \mathrm{srad} \cdot$ year). The exact value depends on the mass composition and will be slightly higher for heavy nuclei than for protons.

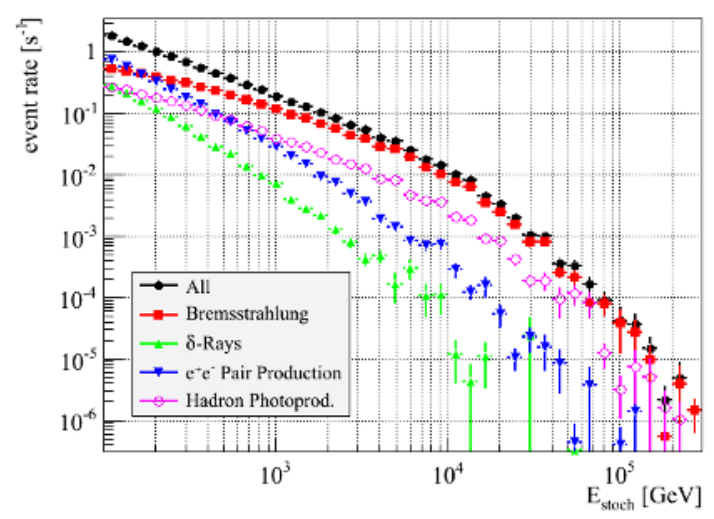

Figure 6. Simulated spectra of all catastrophic losses along muon tracks for IceCube deep detector events by type of interaction as a function of stochastic energy $E_{\text {stoch }}$ CORSIKAgenerated muons were propagated through the ice with the standard IceCube mmc code [13]. Fluxes have been weighted according to the primary flux model proposed by Gaisser [5]. As in the experimental analysis, only events with more than 1,000 total registered photo-electrons are included, corresponding to a single energy loss of about $10 \mathrm{TeV}$. Note that the lower end of the distribution is dominated by cascades occurring in high-multiplicity muon bundles.

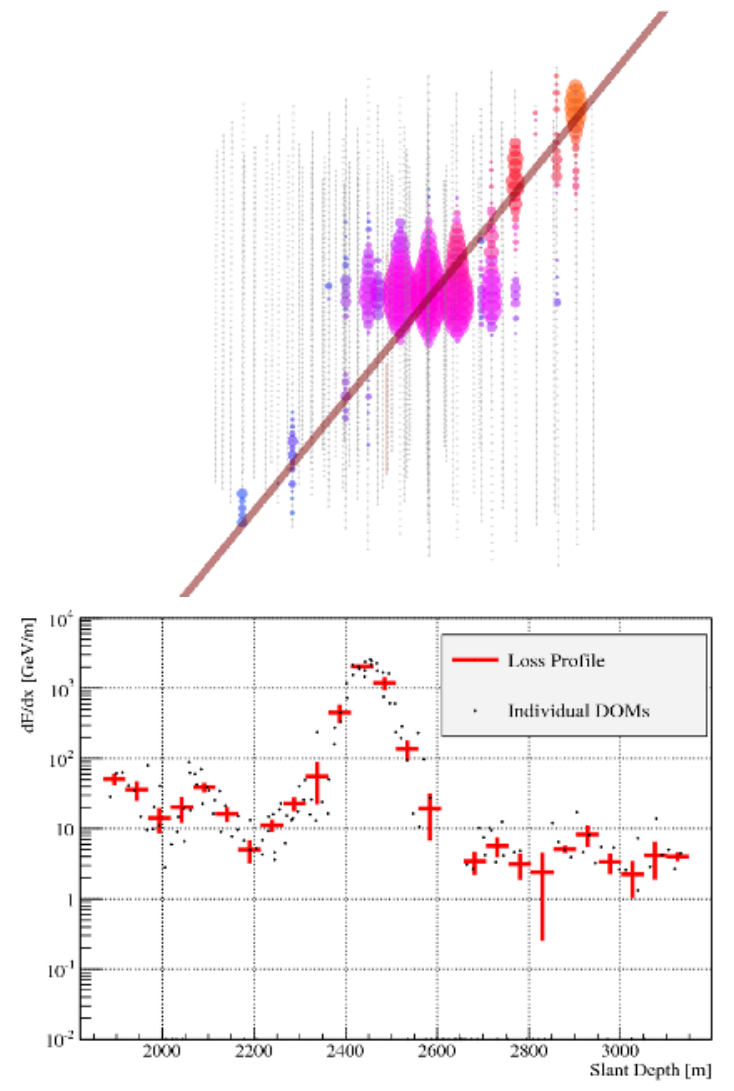

Figure 7. An example of massive stochastic loss event found in IceCube 59-string data. The energy deposition estimate shown in the bottom plot is consistent with an $80 \mathrm{TeV}$ cascade. The ratio of peak over median energy loss is used to identify and tag exceptional stochastic cascades. The $\mathrm{x}$-axis corresponds to the distance along the track, measured from the surface of the ice. For details about the reconstruction method, see [15].

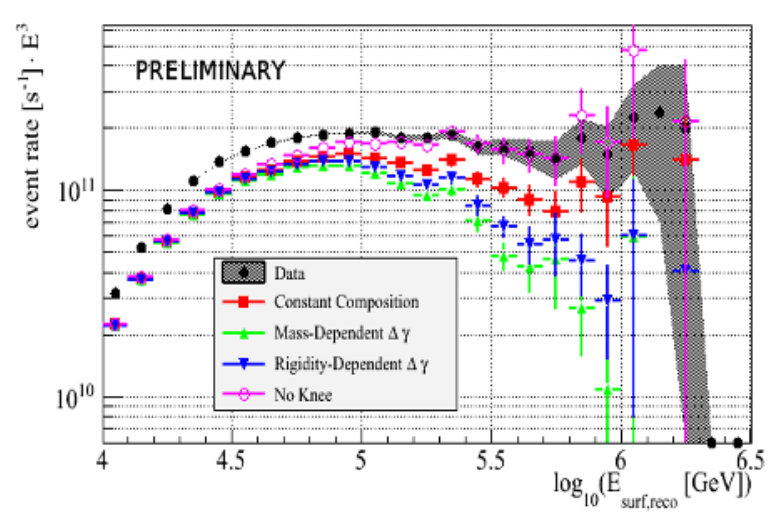

Figure 8. Experimentally measured single muon surface energy $E_{\text {surf,reco }}$ to simulation using data from the IceCube 59-string configuration [15]. The result is compared to three fluxes based on the Poly-Gonato model [11] and a toy model ignoring the cosmic ray knee. As only the conventional muon flux from light mesons was considered in the simulation, the lack of evidence for a cutoff in the spectrum could be due to an emerging prompt component. Errors are statistical only. As can be seen from the size of the error bars, availability of simulated events is a critical factor for the interpretation of the result. 
For astrophysical neutrino searches, precise knowledge of atmospheric lepton production is essential. Since current limits make the detection of neutrino point sources in the near future highly unlikely, the best chance for a discovery currently lies with diffuse searches [12]. For these, neutrinos produced in atmospheric showers form an irreducible background which needs to be carefully modeled before possible excesses due to astrophysical sources can be identified with any confidence. Neglecting to do so may result in lower flux limits, but at the expense of reduced reliability [14].

Atmospheric lepton fluxes in the critical region above $100 \mathrm{TeV}$ depend on the primary cosmic ray composition around the knee and the contribution from prompt hadron decays, in particular from charmed mesons. Both effects are not well understood, as measurements are constrained by instrumental limitations. The cosmic ray flux at these energies is too low for direct detection and forward heavy meson production takes place too close to the beamline to be accessible in collider experiments. Atmospheric muons represent an excellent way to resolve these uncertainties. Compared to neutrinos, statistics are higher and there is no ambiguity about a possible astrophysical origin.

The experimental challenge for a measurement of the single muon spectrum lies in the identification of high-energy muons over a dominant background of highmultiplicity muon bundles, as can be seen from Fig.3. This can be accomplished by tagging exceptionally energetic stochastic losses along muon tracks, which can only be produced by individual muons. An example for a typical data event used in the analysis is shown in Fig. 7. The reconstructed energy of the cascade can then be used to infer the most likely muon energy at the point of production. Details of the analysis procedure can be found in [15].

Figure 6 shows the Monte-Carlo simulated spectra of atmospheric muon-induced cascades in IceCube. Even in a cubic-kilometer scale detector, energy losses above $100 \mathrm{TeV}$ occur only a few times per day. The resulting muon energies can be compared to simulated distributions weighted according to different primary flux models, as demonstrated in Fig. 8. Even though the result is still preliminary, it seems difficult to explain the result with a purely conventional flux from a nucleon spectrum that cuts off at the Knee.

\section{Lateral Muon Distribution}

Muons with high transverse momenta $p_{T}$ produced in air showers carry potentially interesting information about cosmic-ray composition and the relative contributions to the total flux from pions, kaons and heavy hadrons. However, before any conclusions about air shower physics can be made, it first needs to be demonstrated that currently available hadronic interaction models reliably reproduce the underlying processes. For a first evaluation, various measured data distributions from IceCube were simply compared to different simulations with the goal of identifying inconsistencies. The large size of the IceCube detector allows to probe distances from the shower core be-

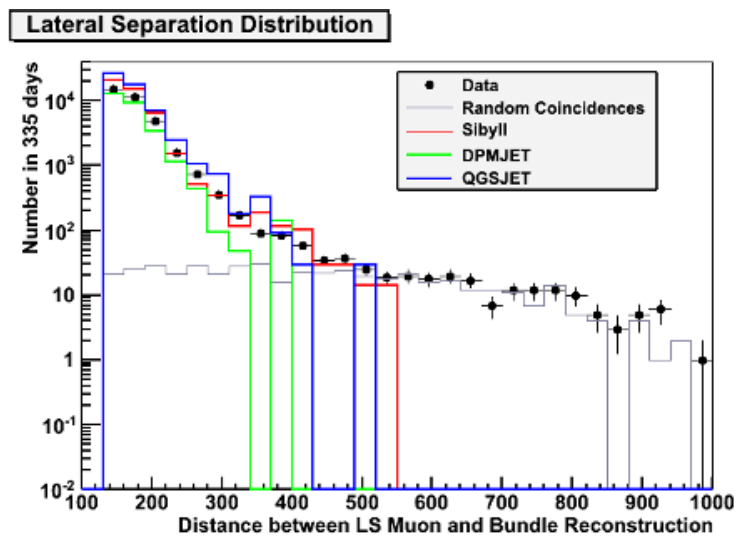

Figure 9. Measured distance between muon bundle and separated muon in IceCube cosmic ray events and CORSIKA simulation [16]. Background due to uncorrelated coincident showers was estimated using off-time events. The distribution shows reasonable agreement with simulated data using different hadronic interaction models.

yond 100 meters, which was not possible in any previous measurement.

The principle of the analysis is the identification of spatially separated muon tracks coincident in time and angle with a bright muon bundle [16]. The main background results from uncorrelated coincident cosmic ray showers and can easily be estimated by searching for parallel muon tracks that would pass the analysis cuts if they were not clearly separated in time. The resulting distribution of lateral distances is shown in Fig. 9.

The exact determination of individual $p_{T}$ values is difficult, as a deconvolution requires a high degree of confidence in the simulation. However, a qualitative description of the underlying physics is possible by simply examining the shape of the distribution as a function of distance from the shower core. Soft interactions producing low transverse momenta cause an exponential behavior, while perturbative QCD predicts a power law for hard interactions.

Fits to the lateral muon distribution indicate that such a transition can be measured in atmospheric showers. Combining a power law with an exponential function results in $\chi^{2} / d o f=45 / 21$, while the purely exponential fit gives $\chi^{2} /$ dof $=68.6 / 21$. The point of transition lies at a separation of about 300 meters, as can be seen in Fig. 10 .

Of particular interest is the zenith angle distribution, as shown in Fig. 11. Clearly, none of the three hadronic models describes the data adequately. Once the cause of this discrepancy is properly understood, it will provide valuable information for future improvements to high-energy air shower simulations.

\section{Cosmic Ray Anisotropy}

Given enough statistics, subtle variations can be detected in the arrival direction of cosmic ray primaries. The unique geographic location of IceCube simplifies the task, as sky coverage does not vary as a function of time. It is also the 


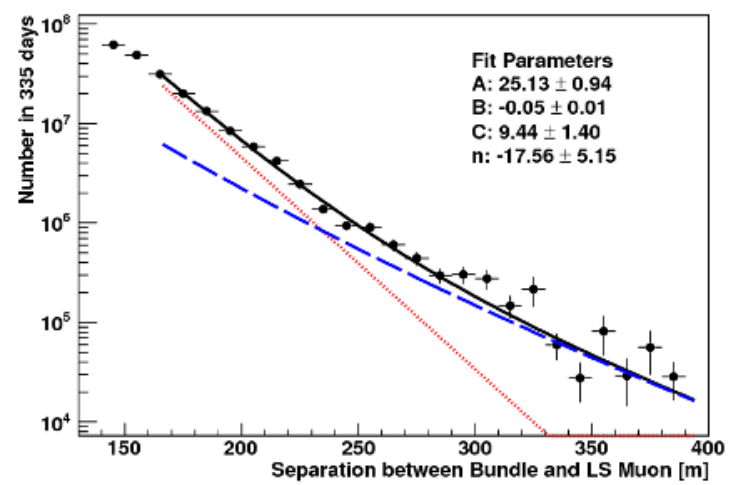

Figure 10. Lateral muon separation at sea level after detector altitude and acceptance correction. The distribution follows an exponential function at low distances. The change to a power law around 300 meters can be interpreted as a transition to the perturbative QCD regime.

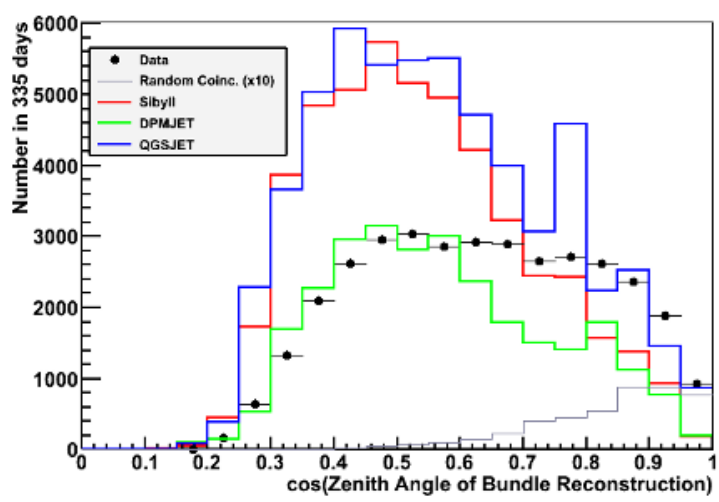

Figure 11. Zenith angle for reconstructed muon bundle track in events containing identifiable laterally separated muons. None of the evaluated hadronic interaction models explains the full shape of the data distribution.

only detector in the Southern Hemisphere that combines a large acceptance area with a low enough threshold to allow measurement of anisotropies at the sub-percent scale, complementing measurements by large air shower arrays on the northern sky.

Already with early detector configurations during the construction phase, it was possible to demonstrate the presence of modulations to the cosmic ray intensity [17]. Variations due to the orbital movement of the Earth around the Sun were in agreement with expectation and validated the experimental approach. The sidereal anisotropy, whose cause is at present still not understood, is shown in Fig. 12 as a function of right ascension.

While the flux anisotropy is clearly dominated by the first two moments, careful analysis of the power spectrum reveals indications for a local maximum at angular scales between 20 and 30 degrees, as can be seen in Fig. 13. Identification of regions that show strong excesses
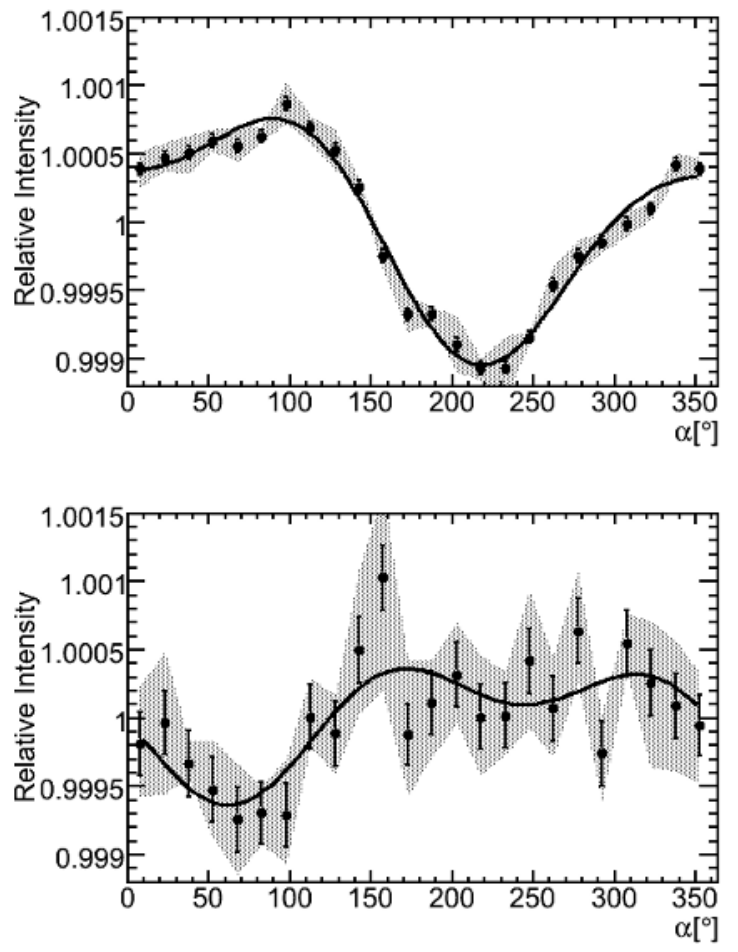

Figure 12. Sidereal modulation of cosmic ray flux measured by IceCube for primaries with median energy of $20 \mathrm{TeV}$ (top) and $400 \mathrm{TeV}$ (bottom) as a function of right ascension. The fit to the data contains a dipole and a quadrupole term. While the amplitude is of comparable magnitude, the phase changes as a function of the energy.

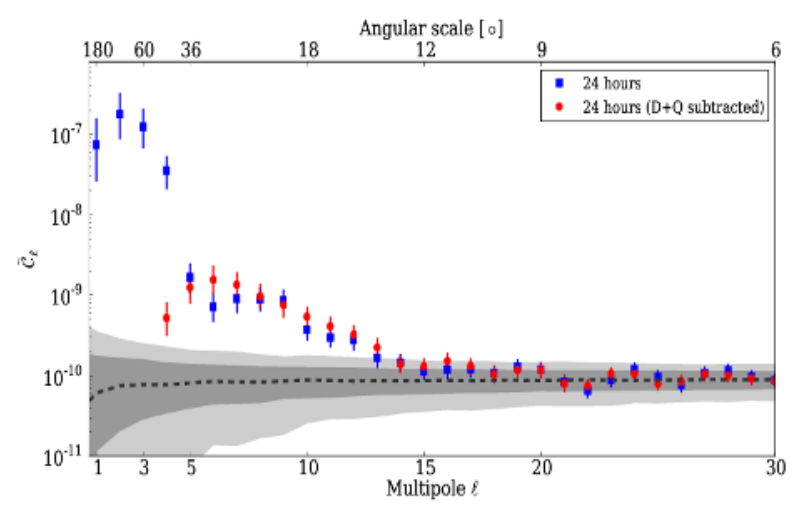

Figure 13. Power spectrum of two-dimensional cosmic ray anisotropy in IceCube data using the 59-string configuration. While the spectrum is dominated by large-scale fluctuations, the data indicates a second peak at angular separations corresponding to higher-order momenta.

or deficits and quantification of the statistical significance will be a main focus for follow-up analyses [18].

An interesting question is whether the anisotropy continues at higher energies. As more statistics become available, the energy range can be extended upwards. Figure 14 shows the preliminary result for the 79-string detector configuration. There is some indication for inhomegeneities 

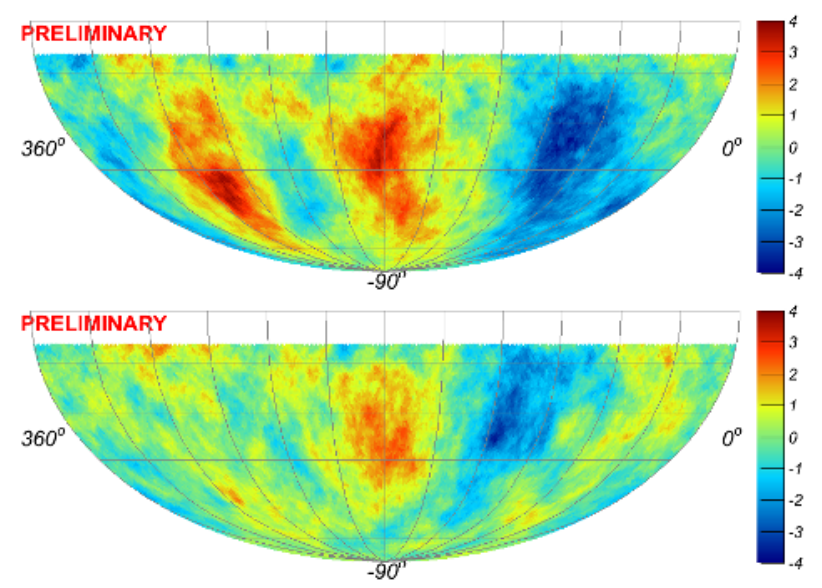

Figure 14. Preliminary sky map for cosmic ray arrival directions in sidereal coordinates using the 79-string configuration. Top: Median primary energy $1 \mathrm{PeV}$. Bottom: Median primary energy $5 \mathrm{PeV}$. The anisotropy at $1 \mathrm{PeV}$ is statistically significant and has been confirmed by the surface array IceTop [19]. Investigation of higher energy ranges will require a larger event sample.

in the arrival direction of cosmic rays up to energies of 5 $\mathrm{PeV}$, but with low statistical significance. Over the coming years, data taken with the full IceCube detector will resolve any ambiguities and extend the scope of the analysis to the knee region and beyond.

\section{Conclusion}

Atmospheric cosmic ray showers are the only astrophysical signal source in IceCube for which positive measurements are guaranteed. Full exploitation of the data set will lead to significant new results in both cosmic ray and particle physics which at the moment are not feasible with any other instrument.

Conceptually simple and robust ways to detect extraterrestrial neutrino sources such as point source analyses have led to limits that make a detection unlikely within the next few years. On the other hand, searches for diffuse astrophysical neutrinos appear to show tenuous indications for a possible excess over the atmospheric flux. Cosmic ray physics will accordingly increase in importance within the IceCube collaboration, since its thorough understanding is essential for a proper interpretation of the neutrino energy spectrum. While for a long time the atmospheric lepton flux, particularly the muon component, has been dismissed as undesirable background, it now becomes apparent that it is an important signal in its own right.

\section{References}

[1] Francis Halzen and Spencer R. Klein, Review of Scientific Instruments 81081101 (2010).

[2] IceCube Collaboration: R. Abbasi et al., accepted by Nuclear Instruments and Methods, arXiv:1207.6326

[3] P. Desiati and T. K. Gaisser, Phys. Rev. Lett. 105, 121102 (2010).

[4] T. K. Gaisser, Cosmic Ray and Particle Physics (Cambridge University Press, 1990).

[5] T. K. Gaisser, Astropart. Phys. 35, 801 (2012).

[6] IceCube Collaboration: R. Abbasi et al., Nuclear Instruments and Methods A618, 139-152 (2010).

[7] IceCube Collaboration: R. Abbasi et al., Nuclear Instruments and Methods A601, 294-316 (2009).

[8] J. Blumer, R. Engel and J. R. Horandel, Prog. Part. Nucl. Phys. 63, 293 (2009). [arXiv:0904.0725 [astroph.HE]].

[9] The KASCADE-Grande Collaboration: W. D. Apel et al., accepted by Astroparticle Physics, arXiv:1206.3834.

[10] S. F. Berezhnev et al., Nucl. Instrum. Meth. A 692 , 98 (2012).

[11] J. R. Hoerandel, Astropart. Phys. 19, 193 (2003).

[12] See e.g. the contributions by J. Brunner and E. Middell in these Proceedings.

[13] D. Chirkin and W. Rhode, arXiv:hep-ph/0407075.

[14] IceCube Collaboration: R. Abbasi et al., Physical Review D84, 082001 (2011).

[15] P. Berghaus and C. Xu for the IceCube Collaboration, Proceeding of the 32nd ICRC, arXiv:1111.2735.

[16] IceCube Collaboration: R. Abbasi et al., accepted by PRD, arXiv:1208.2979.

[17] IceCube Collaboration: R. Abbasi et al., Astrophysical Journal 746, 33 (2012).

[18] IceCube Collaboration: R. Abbasi et al., Astrophysical Journal 740, 16 (2011).

[19] IceCube Collaboration: M. G. Aartsen et al, submitted to ApJ, arXiv:1210.5278. 